\title{
Elements Related to Heterogeneity of Antibody-Dependent Cell Cytotoxicity in Patients Under Trastuzumab Therapy for Primary Operable Breast Cancer Overexpressing Her2
}

\author{
Stefania Varchetta, ${ }^{1}$ Nadia Gibelli, ${ }^{1}$ Barbara Oliviero, ${ }^{1}$ Elena Nardini, ${ }^{2}$ Roberto Gennari, \\ Giovanna Gatti, ${ }^{4}$ Luzemira Santos Silva, ${ }^{4}$ Laura Villani, ${ }^{1}$ Elda Tagliabue, \\ Sylvie Ménard, ${ }^{2}$ Alberto Costa, ${ }^{1}$ and Francesco F. Fagnoni ${ }^{1}$
}

${ }^{1}$ Oncology Department, Scientific Institute of Pavia, Fondazione Salvatore Maugeri-Clinica del Lavoro e della Riabilitazione, Istituti di Ricovero e Cura a Carattere Scientifico, Pavia, Italy; ${ }^{2}$ Department of Experimental Oncology, Fondazione Istituto Nazionale per lo Studio e la Cura dei Tumori; ${ }^{3}$ European School of Oncology; and ${ }^{4}$ Department of Surgery, Istituto Europeo di Oncologia, Milan, Italy

\begin{abstract}
Preliminary results from a pilot trial on trastuzumab's mechanism of action against operable breast tumors overexpressing Her2 suggested a role for antibody-dependent cell cytotoxicity (ADCC). To examine factors affecting ADCC intensity and variability, we extended this study to the phenotypic and functional analysis of circulating mononuclear cells in 18 patients. ADCC was induced by trastuzumab therapy in 15 of 18 patients $(83 \%)$. Inability to develop ADCC in three patients did not depend on inadequate levels of trastuzumab because further increase in its concentration in vitro was ineffective. Rather, susceptibility to develop ADCC was fairly predicted by test with trastuzumab before therapy and was correlated to the number of lymphocytes coexpressing CD16 and CD56. Phenotypic analysis at the end of ADCC evaluating down-regulation of CD16, and up-regulation of CD69 and CD107a, confirmed that natural killer (NK) cells and $\mathrm{CD56}^{+} \mathrm{T}$ cells were involved in productive engagement of trastuzumab. Also, the killing efficiency of $\mathrm{CD}^{+} 6^{+}$lymphocytes was influenced by $158 \mathrm{~V} / \mathrm{F}$ polymorphism of FcyRIII (CD16), whereas variations of CD247 on NK cells were consistent with trends between ADCC before and after therapy. Complete pathologic response was observed in one patient showing ADCC of outstanding intensity, whereas four cases of partial response showed intermediate ADCC; none of the three patients unable to mount ADCC had significant tumor regression. These data indicate that quantity and lytic efficiency of $\mathrm{CD}^{+} 6^{+}$lymphocytes are major factors for ADCC induction by trastuzumab, and confirm that breast cancer responses to short-term trastuzumab monotherapy may depend on involvement of the ADCC mechanism. [Cancer Res 2007;67(24):11991-9]
\end{abstract}

\section{Introduction}

Clinical studies have shown that trastuzumab can induce regression of breast cancer (1-5). However, even when patients are rigorously preselected on the basis of Her2 antigen expression, not all of them respond to trastuzumab. Indeed, a significant percentage of patients shows intrinsic primary resistance to

\footnotetext{
Note: N. Gibelli and B. Oliviero contributed equally to this work.

Requests for reprints: Francesco F. Fagnoni, Experimental Oncology Laboratory, Fondazione Salvatore Maugeri-Istituti di Ricovero e Cura a Carattere ScientificoClinica del Lavoro e della Riabilitazione, via S. Maugeri, 10, 27100 Pavia, Italy. Phone: 39-0382-592062; Fax: 39-0382-592061; E-mail: francesco.fagnoni@fsm.it.

(C)2007 American Association for Cancer Research.

doi:10.1158/0008-5472.CAN-07-2068
}

trastuzumab, although the causes of such resistance are currently unclear (6).

Clinical efficacy of trastuzumab has been postulated to depend on multiple direct or indirect action mechanism that may produce cytostatic and/or cytotoxic antitumor cell effects (7). Direct binding can induce Her-2 down-regulation and alteration of several Her2-dependent cellular signaling pathways with either cytostatic or cytotoxic antitumor effects. On the contrary, indirect mechanisms are referred to triggering of immune responses after trastuzumab engagement through Fc-receptors expressed by leukocytes, and they are thought to have mainly cytotoxic antitumor effects. Accordingly, studies in animal models have shown that a substantial contribution to cytotoxic trastuzumabinduced regression of xenotransplanted tumors is provided by host immune mechanisms (8), but their role in clinical activity is still controversial. Among others, antibody-dependent cell cytotoxicity (ADCC) is an immune effector mechanism that induces indirect tumor cell killing very efficiently and, in so doing, may also mediate cytocidal tumor regression caused by trastuzumab and other mAbs. However, evaluation of ADCC in metastatic breast cancer patients treated with a combination of trastuzumab and interleukin-2 (IL-2) or IL-12 did not confirm a correlation with clinical responses (9-11). However, later studies in early-stage breast cancer treated before surgery with trastuzumab as a single agent reported data suggesting a role for ADCC. In particular, we have found that patients induced to complete or partial remission by trastuzumab alone were found to have higher ADCC and higher in situ infiltration of cytotoxic lymphocytes, including natural killer (NK) cells (12). Likewise, even when used in combination with docetaxel, neoadjuvant trastuzumab treatment was found to be associated with significantly increased numbers of tumorassociated NK cells and increased lymphocyte expression of cytotoxic activity markers compared with controls (13). Moreover, a study on the action mechanism of trastuzumab in the neoadjuvant setting confirmed that tumor regression is dependent on a cytocidal rather than cytostatic antitumor effect and suggested that this effect is mediated by programmed cell death (apoptosis; ref. 5). Interestingly, this latter evidence is not inconsistent with a supposed role for ADCC because apoptosis may also represent the final common death pathway for ADCC-mediated cytotoxicity (14).

When assuming that ADCC may constitute a mechanism involved in trastuzumab activity, its efficacy may depend on both humoral and cellular factors. Humoral factors such as plasma monoclonal antibody $(\mathrm{mAb})$ concentration is thought to be critical not only in absolute terms but also in relative terms of competition with autologous IgG1 immunoglobulins for binding to low-affinity 
Fc- $\gamma$ receptor type III (CD16; ref. 15). Similarly, the array of cellular effectors potentially exerting ADCC includes not only $\mathrm{CD} 3^{-} \mathrm{CD} 56^{+}$ NK lymphocytes (16), but also $\mathrm{CD}^{+}{ }^{+} \mathrm{CD} 16^{+}$T-cell subset (17), $\mathrm{CD}_{16}{ }^{+} \mathrm{CD} 33^{+}$macrophages (18), and $\mathrm{CD}^{+} 6^{+}$granulocytes (19). Finally, the Fc-receptor binding affinity for IgG1 is diverged by genetic polymorphism such as CD16-158V/F $(20,21)$. Indeed, a higher affinity of CD16-158V/V has been reported to correlate with improved clinical responses observed after IgGl mAb immunotherapy in some instances $(22-24)$ but not in others $(25,26)$. On the whole, ADCC can be considered as the sum of several factors; however, it remains to be determined which is the specific contribution of each of these in individual patients with an established disease once therapy is under way and possibly without the immunosuppressive effects of other drugs.

In this study, we have analyzed the intensity of $\mathrm{ADCC}$ in patients with primary operable breast tumors overexpressing HER2 before and after short-term preoperative therapy with trastuzumab as single agent. We found broad individual variability of ADCC levels that was significantly related to the number of $\mathrm{CD}^{+} 6^{+} \mathrm{CD} 56^{+}$ effector lymphocytes, but not to trastuzumab serum levels. Such variability was confirmed to correlate with trastuzumab clinical activity especially at the upper and lower limits of ADCC activity. These data can provide potentially useful insights for understanding the causes of intrinsic primary resistance to trastuzumab and increase the efficacy of short-term trastuzumab-based treatments.

\section{Materials and Methods}

Patients. From May 2002 to June 2005, 18 patients with primary operable breast cancer classified as HER2-positive tumors (herceptest $/ 3^{+}$or herceptest $/ 2^{+}$along with positive fluorescence in situ hybridization testing) on the basis of core biopsy pathologic analysis were enrolled in a study protocol of preoperative trastuzumab therapy as a single agent, as previously described elsewhere (12). The study protocol was approved by the institutional review board, and patients were enrolled after obtaining informed consent. After completion of clinical assessment and core biopsy evaluation, patients received preoperative trastuzumab (Herceptin, kindly provided by Roche S.p.A.) as a single loading dose of $4 \mathrm{mg} / \mathrm{kg}$, followed by three weekly treatments at $2 \mathrm{mg} / \mathrm{kg}$. Left ventricular ejection fraction was determined at baseline and before surgical treatment. Responses to trastuzumab therapy were evaluated based on a clinical, pathologic, and bidimensional radiologic/ultrasound examination of the tumor before and after treatment, and were classified according to Response Evaluation Criteria in Solid Tumors (27). Surgery was performed 1 week after the last dose. After surgery, patients received adjuvant treatment in accordance with standard practice guidelines. Blood samples were collected in all patients immediately before beginning the trastuzumab therapy and 1 week after the last treatment.

Isolation of patients' sera and peripheral blood mononuclear cells. Two distinct aliquots of peripheral blood, without and with anticoagulant, were drawn from patients before and after therapy with trastuzumab. Peripheral blood collected without anticoagulant was used for separation of autologous serum, which was further incubated for $30 \mathrm{~min}$ at $56^{\circ} \mathrm{C}$ to inactivate complement; the separated serum was finally frozen at $-80^{\circ} \mathrm{C}$ until use. Concomitantly, the peripheral blood collected in the anticoagulant was used to isolate peripheral blood mononuclear cells (PBMC) by standard Ficoll gradient centrifugation (Ficoll-Paque, Amersham Pharmacia Biotech). After centrifugation, PBMCs were washed with a medium containing $10 \%$ heat-inactivated fetal bovine serum (FBS), resuspended in $90 \%$ FBS $+10 \%$ DMSO solution on ice at a cell concentration $<10 \times 10^{6} / \mathrm{mL}$ and, lastly, frozen in liquid nitrogen after intermediate cooling at $-80^{\circ} \mathrm{C}$ in a methanol-containing device. The viability of PBMCs after thawing was consistently higher than $85 \%$ to $90 \%$, as evaluated by flow cytometry with standard propidium iodide incorporation.
Evaluation of patients' ADCC. The objective to reestablish conditions in vitro as close as possible to those in vivo was pursued by testing PBMC against an Her2-overexpressing target cell line in the presence of simultaneously harvested autologous serum. To obtain a reliable comparison between ADCC before and after therapy, PBMCs isolated from the same patient, but at different times, were thawed and tested in parallel in the same experiment. ADCC by trastuzumab was measured in a shortterm ${ }^{51} \mathrm{Cr}$ release assay using radiolabeled target cells from the Her2overexpressing MDA-MB361 human breast cancer cell line. To this end, 1 million MDA-MB361 target cells in exponential growth were labeled with $100 \mu \mathrm{Ci}(3.7 \mathrm{MBq})$ of $\mathrm{Na}^{51} \mathrm{Cr}$ (Amersham International) for $1 \mathrm{~h}$ at $37^{\circ} \mathrm{C}$, washed extensively, and used as targets. Five thousand ${ }^{51} \mathrm{Cr}$-labeled target cells per well were seeded into 96-well U-bottomed plates. Experiments were conducted in triplicates at various PBMC (effector) to MDA-MB361 (target) ratios of 6:1; 12.5:1; 25:1, and 50:1, in $200 \mu \mathrm{L}$ of RPMI 1640 containing $20 \%$ autologous human serum. The antibody source for evaluation of patients' ADCC was made up, respectively, of Herceptin (same as for clinical use, aliquoted in saline and stored as working solution; kindly provided by Roche), autologous serum of treated patients, or both. Experiments conducted in a preliminary phase to select optimal conditions for the antibody-dependent cytotoxicity effect showed that $(a)$ target cells were not killed after exposure to trastuzumab or serum in the absence of PBMC effectors; $(b)$ the coating of effectors with trastuzumab followed by the removal of soluble $\mathrm{mAb}$ in excess provided negligible target cell killing; and (c) the coating of target cells with trastuzumab followed by the removal of soluble mAb in excess contributed only limited target cells killing, whereas (d) the persistence of soluble trastuzumab during the assay not only reproduced more closely the in vivo situation but also represented the condition providing the highest killing of target cells (data not shown). When testing for different trastuzumab concentrations as well as different concentrations of autologous serum, we found that either 1 to $10 \mu \mathrm{g} / \mathrm{mL}$ of trastuzumab or $10 \%$ to $20 \%$ of posttherapy patients' sera were optimal sources for saturating ADCC assay by PBMC (data not shown). Thus, all ADCC experiments were carried out in the presence of $20 \%$ autologous serum, without or with $10 \mu \mathrm{g} / \mathrm{mL}$ of trastuzumab added exogenously. After $4 \mathrm{~h}$ at $37^{\circ} \mathrm{C}$ in humidified air with $8 \% \mathrm{CO}_{2}$, the release of ${ }^{51} \mathrm{Cr}$ into supernatants was determined in Lumaplate 96 scintillation plates, and counted by a TopCount gamma counter (Packard Instruments Company). Maximal and spontaneous ${ }^{51} \mathrm{Cr}$ release values were obtained by adding either 1\% NP40 detergent (BDH Biochemicals) or complete medium, respectively, to microtiter wells containing $5 \times 10^{3}$ labeled target cells. The percentage of lysis was calculated according to the standard formula $=$ $100 \times(\mathrm{cpm}$ experimental $-\mathrm{cpm}$ spontaneous release $) /(\mathrm{cpm}$ maximum release $-\mathrm{cpm}$ spontaneous release).

Flow cytometry analysis of PBMC effectors for ADCC. A sixparameter flow cytometric analysis of thawed PBMC was performed using a two-laser FACSCalibur instrument equipped with CellQuest software (BD Biosciences). Instrument setting and compensation were established as described previously (28). Data acquisition was always done immediately after staining procedures. To identify phenotype modifications of effector lymphocytes involved in trastuzumab-mediated cytotoxicity, $4 \times 10^{5}$ of the same thawed PBMCs prepared for use in ADCC assay were left in parallel cultures with unlabeled MDA-MB361 target cells at an effector-to-target ratio of 25:1 in the absence (negative control) or presence of trastuzumab $(10 \mu \mathrm{g} / \mathrm{mL})$, in otherwise identical culture conditions of ADCC assay. A further fraction of such cultures was dedicated to testing of CD107a mobilization by adding $1 \mu \mathrm{g} / 10^{6}$ cells of anti-CD107a FITC-conjugated (mouse IgG1, H4A3, BD PharMingen) at the beginning of culture. At the end of culture, cells were harvested, washed, and directly stained with $1 \mu \mathrm{g} / 10^{6}$ cells of each of the following mAbs: anti-CD3 conjugated to allophycocyanin (UCHT1, mouse IgG1, BD PharMingen), anti-CD56 conjugated to Rphycoerythrin cyanin 5.1 (N901, mouse IgG1, Beckman-Coulter), antiCD16 conjugated to R-phycoerythrin (3G8, mouse IgG1, BD PharMingen), and anti-CD69 conjugated to FITC (FN50, mouse IgG1, BD PharMingen), or with isotype-matched mAbs of irrelevant specificity R-phycoerythrin- and FITC-conjugated as negative controls for anti-CD16 and anti-CD69, respectively. To identify the frequency of effector NK and $\mathrm{T}$ populations 
among PBMC submitted to ADCC assay, an aliquot of the same thawed PBMC prepared for use as effectors in ADCC were washed in calciummagnesium-free PBS containing 5\% FBS and mouse serum, and directly stained with mAbs anti-CD3 (allophycocyanin), anti-CD56 (PC5), and antiCD16 (R-phycoerythrin). For evaluation of CD247 (TcR $\zeta$-chain) expression intensity on $\mathrm{T}$ and NK cells, PBMC freshly isolated by Ficoll gradient after venipunctures obtained from eight patients (from patient 11 through patient 18) were submitted for direct surface staining with anti-CD3 plus anti-CD56, washed, fixed, and permeabilized (Fix\&Perm, Caltag Laboratories), and incubated either with anti-CD247 (MCA1297F, mouse IgG2a, FITC-conjugated; Serotec), or a mouse IgG2a-FITC mAbs of irrelevant specificity (negative control). The expression intensity of CD247, defined separately on T and NK cells, was obtained as the ratio between mean fluorescence intensity of anti-CD247 and that of its negative control, as described elsewhere (29).

Characterization of Fc $\gamma$ RIIIA-158 V/F polymorphism. The FCR $\gamma$ IIIA$158 \mathrm{~V} / \mathrm{F}$ polymorphism was determined by two methods: at the genotypic level in the first 10 recruited patients and at the phenotypic level in all 18 patients. The $158 \mathrm{~V} / \mathrm{F}$ genetic polymorphism of $F C G R 3 A$ was characterized on genomic DNA extracted from thawed PBMC using minor modifications of standard procedures for peripheral blood. Genotyping of Fc $\gamma \mathrm{R} 3 \mathrm{~A}-158 \mathrm{~V} / \mathrm{F}$ polymorphism was performed using a nested PCR followed by allele-specific restriction enzyme digestion. Two specific Fc $\gamma$ R3A primers (5-ATATTTACAGAATGGCACAGG-3 and 5-GACTTGGTACCCAGGTTGAA-3) were used to amplify a 1.2-kb fragment containing the polymorphic site. The initial PCR assay was performed with $1.25 \mu \mathrm{g}$ genomic DNA, $200 \mathrm{ng}$ of each primer, $200 \mu \mathrm{mol} / \mathrm{L}$ of each deoxyribonucleoside triphosphate (dNTP), $1 \mu \mathrm{mol} / \mathrm{L}$ $\mathrm{MgCl}_{2}$, and 1 unit Taq DNA polymerase. This first PCR consisted of $10 \mathrm{~min}$ at $95^{\circ} \mathrm{C}$, then 35 cycles (each consisting of steps at $95^{\circ} \mathrm{C}$ for $1 \mathrm{~min}, 57^{\circ} \mathrm{C}$ for $1.5 \mathrm{~min}$, and $72^{\circ} \mathrm{C}$ for 1.5 minutes), and $8 \mathrm{~min}$ at $72^{\circ} \mathrm{C}$ to achieve complete extension. The second PCR used primers (5-ATCAGATTCGATCCTACTTCTGCAGGGGGCAT-3; 5-ACGTGCTGAGCTTGAGTGATGGTGATGTTCAC-3) amplifying a 94-bp fragment and creating a NlaIII restriction site only in the FcyR3A-158V allele. This nested PCR was performed with $1 \mu \mathrm{L}$ of the amplified DNA, $150 \mathrm{ng}$ of each primer, $200 \mu \mathrm{mol} / \mathrm{L}$ of each dNTP, $1.5 \mu \mathrm{mol} / \mathrm{L} \mathrm{MgCl}_{2}$, and 2.5 units of Taq DNA polymerase. The first cycle consisted of $5 \mathrm{~min}$ at $95^{\circ} \mathrm{C}$, then 35 cycles (each consisting of steps at $95^{\circ} \mathrm{C}$ for $1 \mathrm{~min}, 62^{\circ} \mathrm{C}$ for $1 \mathrm{~min}$, and $72^{\circ} \mathrm{C}$ for $1 \mathrm{~min}$ ), and $9.5 \mathrm{~min}$ at $72^{\circ} \mathrm{C}$ to complete extension. The amplified DNA $(10 \mu \mathrm{L})$ was then digested with 10 units $\mathrm{Nla \textrm {III }}$ at $37^{\circ} \mathrm{C}$ for $12 \mathrm{~h}$ and separated by electrophoresis on $8 \%$ polyacrylamide gel. After staining with ethidium bromide, DNA bands were visualized under UV light. For homozygous Fc $\gamma R 3 \mathrm{~A}-158 \mathrm{~F}$ patients, only one undigested band ( $94 \mathrm{bp}$ ) was visible. Three bands (94, 61, and $33 \mathrm{bp}$ ) were seen in heterozygous individuals, whereas for homozygous Fc $\gamma$ R3A-158V patients only two digested bands (61 and $33 \mathrm{bp}$ ) were obtained.

Evaluation of FCR $\gamma$ IIIA-158V/F phenotypic polymorphism was done in all patients on PBMC by using flow cytometry assay described by Bottcher et al. (30) by two anti-FCR $\gamma$ IIIA mAbs: 3G8 (mouse IgG1, FITC-conjugated, BD PharMingen) and MEM-154 (mouse IgGl, FITC-conjugated, Immunological Sciences). As reported, 3G8 mAb binds to a nonpolymorphic epitope of FCR $\gamma$ IIIA with invariable affinity and regardless of $158-\mathrm{F} / \mathrm{V}$ polymorphism, whereas the binding of MEM-154 mAb to its epitope on FCR $\gamma$ IIIA is dependent on valine amino acid expression at $158-\mathrm{F} / \mathrm{V}$. The differential binding of the two mAbs used in parallel, expressed as the ratio between the mean fluorescence intensities of their conjugated fluorochromes, allows to distinguish three different phenotypes: $\mathrm{F} / \mathrm{F}$ (MEM-154/3G8 ratio $<0.04$ ), V/V (ratio $>0.62$ ), and $\mathrm{F} / \mathrm{V}$ (ratio between 0.15 and 0.48 ). The accordance between results on FCR $\gamma$ IIIA- $158 \mathrm{~V} / \mathrm{F}$ polymorphism obtained separately by the genotypic and phenotypic methods in the first 10 patients was absolute. Patients 11 to 18 were characterized by flow cytometry alone.

Statistical analysis. To compare ADCC of each patient observed at different times or with versus without trastuzumab, paired analysis was performed using Student's $t$ test. Nonparametric Wilcoxon rank sum test was used to compare data between groups. Correlations were analyzed by Spearman's correlation coefficient test. Statistical tests were performed with Stat-View software, and $P<0.05$ was considered significant.

\section{Results}

Analysis of trastuzumab-dependent ADCC in patients with operable breast cancer overexpressing Her2. The first objective was to evaluate the effect of trastuzumab therapy on ADCC in patients having effector cells not altered by any other previous therapy. As shown in Fig. $1 A$, ADCC activity exerted by PBMC in the presence of autologous sera increased significantly after 4 weeks of therapy with trastuzumab in 15 of $18(83 \%)$ patients enrolled in the study. Basal ADCC activity before therapy was negligible $(<10 \%)$ in all patients but one. Patient 1 showed not only high spontaneous cytotoxic activity but also maximal response after therapy. Most other patients induced intermediate lysis of target cells. In the remaining three cases (patients 4,8 , and 18), no significant increase was induced after therapy. Overall, the increase was significant when considering collectively all patients $(P<0.001$, Fig. $1 A$, right), thus confirming that short-term therapy with trastuzumab was active at inducing ADCC activity. However, given the wide variability ranging from maximal response (one patient) to unresponsiveness (three patients), these data also indicated that the susceptibility to developing ADCC was not universal and highly heterogeneous.

The second objective was to evaluate the distinct contribution of trastuzumab-containing serum to ADCC heterogeneity to clarify whether low responses could be caused by inadequate availability of trastuzumab after therapy. To this end, we conducted parallel assays with saturated concentration in vitro. As shown in Fig. $1 B$, results obtained with autologous sera after therapy alone and with further addition of trastuzumab were highly correlated and definitely overlapping. Similarly, the ADCC activity of PBMC from poorly responsive patients could not be restored by adding serum from the patient with the highest ADCC response (not shown). Conversely, when we tested PBMC from patients after therapy in the presence of medium supplemented only with FBS or heterologous human serum from untreated donors, ADCC activity was abolished (not shown). These data confirmed the essential role of trastuzumab in conferring activity in ADCC assay, but ruled out the possibility that either unresponsiveness or low responses could depend on inadequate drug pharmacokinetic.

Thereafter, we asked whether individual potential for trastuzumab-dependent ADCC might be constitutive to each patient, thus preexisting the therapy and possibly predicted by testing PBMC before therapy in the presence of saturated amounts of trastuzumab. As shown in Fig. $1 C$, the equivalence between values observed before therapy and those observed after therapy was not absolute but still significant. This correlation suggests that this individual characteristic can be roughly predicted at least on a short-term basis and can possibly distinguish high from intermediate and low ADCC responders.

Analysis of cellular and molecular variables affecting individual variability of trastuzumab-dependent ADCC. To identify effector cells responsible for trastuzumab-dependent short-term responses, we analyzed the phenotype of lymphocytes cultured for ADCC assay. We focused the analysis on $\mathrm{CD}^{+} 6^{+} \mathrm{CD} 3^{-}$ $\mathrm{NK}$ cells, $\mathrm{CD}^{+} \mathrm{CD}^{+} 6^{+} \mathrm{T}$ cells, and $\mathrm{CD}^{+} \mathrm{CD} 56^{-} \mathrm{T}$ cells (Fig. 2, first row from the top). The $\mathrm{CD}^{+} 6^{+} \mathrm{CD} 3^{-} \mathrm{NK}$ cell population showed a remarkable down-modulation of $\mathrm{CD} 16$ in the presence of target cells and trastuzumab (Fig. 2, second row, thick lines). Such down-modulation was specific for productive engagement of CD16 by trastuzumab, because it was not observed when PBMC were cultured in the presence of either target cells (thin lines) or trastuzumab alone (not shown). Similarly, NK cells also showed 


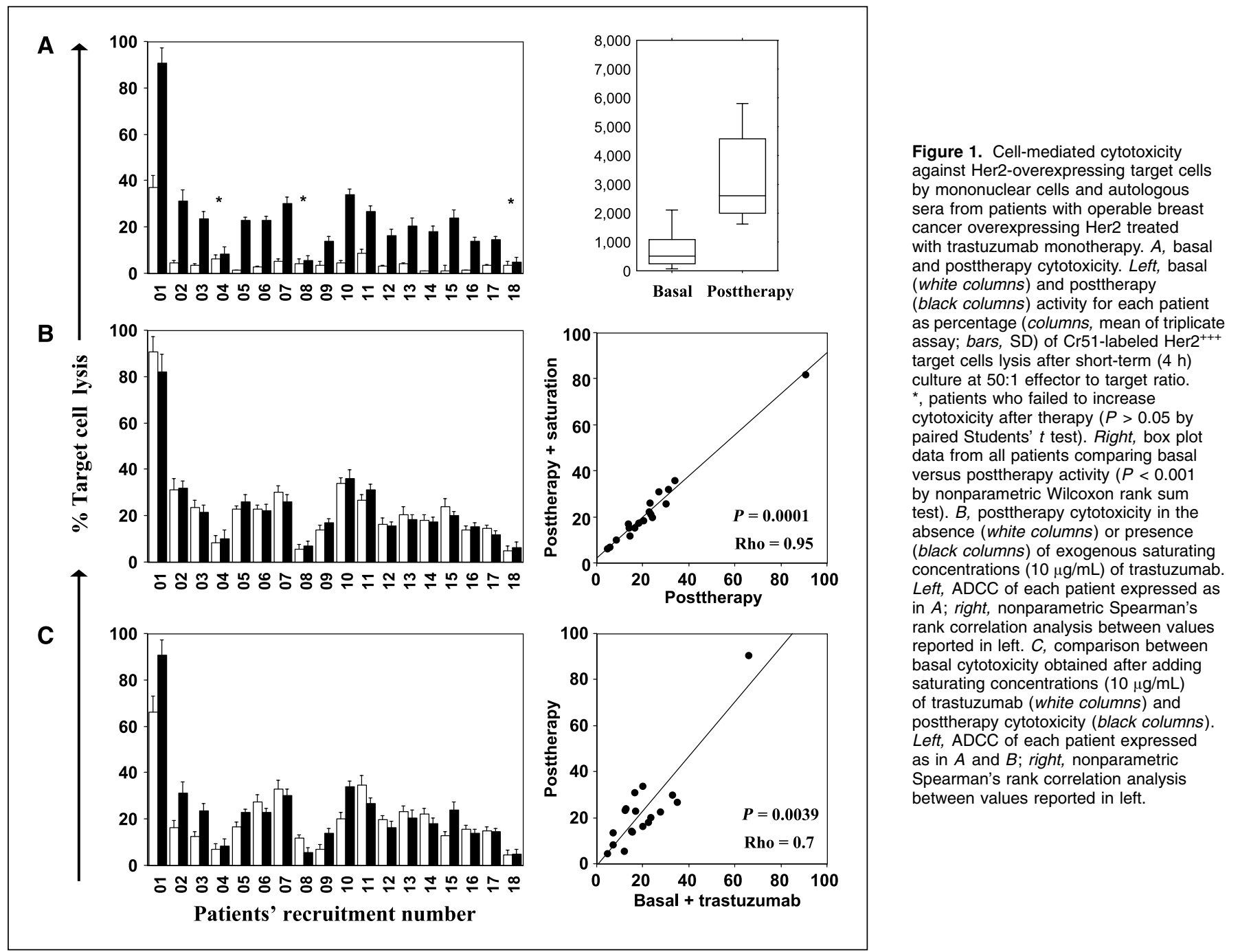

specific up-regulation of CD69 early activation antigen (Fig. 2, third row) and expression of CD107a (Fig. 2, fourth row), a molecule linked to the killing activity of cytotoxic lymphocytes. The $\mathrm{CD}^{+} \mathrm{CD} 6^{+}$T-cell subset also showed similar phenotypic modifications, but to a lesser extent. By contrast, $\mathrm{CD}^{+} \mathrm{CD} 56^{-}$ $\mathrm{T}$ cells showed no expression of CD16 and did not up-regulate either CD69 or CD107a. Therefore, we hypothesized that patients' ADCC heterogeneity might have been related to the proportion of putative effector lymphocytes constituted by NK and T cells coexpressing CD16 and CD56. The most evident correlation between target lysis and effector lymphocytes was found in patient 1 (Fig. 3, first row from the top), who revealed coexisting outstanding levels of ADCC and effector lymphocytes. Nevertheless, other patients with intermediate and low ADCC levels also had intermediate and low quantity of effectors, respectively (Fig. 3, second and third row). The correlation between percentages of $\mathrm{CD}^{+} 6^{+} \mathrm{CD}^{+} 6^{+}$effectors in PBMC used for ADCC and ADCC itself was statistically significant before and, although to a lesser degree, also after therapy (Fig. $4 B$ ). On the whole, these findings confirmed that $\mathrm{NK}$ and $\mathrm{T}$ cells expressing $\mathrm{CD} 16$ constitute the effector lymphocytes mainly involved in short-term trastuzumabdependent cytotoxicity, and their variability explained, at least partially, the patients' ADCC heterogeneity.
To gain further insights into other factors possibly affecting individual variability, we explored the potential involvement of CD16 V/F polymorphism at position 158. Patients were found to be distributed unequally among the three allelic groups: VV (three patients only), VF (patient 10), and FF (patient 5; Table 1). When we searched for differences between ADCC levels of the three groups, we found no differences (not shown) probably because the quantity of effector $\mathrm{CD} 16^{+} \mathrm{CD} 56^{+}$lymphocytes was not consistent with CD16 polymorphism. By contrast, when target cell lysis (ADCC) data were normalized for a fixed number of effector cells, we found some preliminary evidence that the killing efficiency of $\mathrm{CD} 16^{+} \mathrm{CD} 56^{+}$cells in the presence of trastuzumab and sera of patients bearing VV alleles was higher than those with VF and FF alleles (Fig. 4C). However, such a relationship between CD16-158V/ F polymorphism and the killing efficiency of $\mathrm{CD} 6^{+} \mathrm{CD} 56^{+}$cells was somewhat weaker in the same patients after therapy (not shown). This latter inconsistency that emerged between observations made before and after therapy added to those previously mentioned about the weakened relationships between $\mathrm{ADCC}$ and $\mathrm{CD} 16^{+} \mathrm{CD} 56^{+}$ effectors (Fig. $4 A$ and $B$ ). Thus, we examined some parameters that could contribute to explaining ADCC alterations occurring with therapies, such as the number of accessory myeloid cells in PBMC, the expression on NK cells and $\mathrm{CD}^{+} 6^{+} \mathrm{T}$ cells of short- and 
long-term activation markers, of perforin content, and the expression of CD247 (the $\zeta$-chain for signal transduction of T-cell receptor and CD16). None of these parameters revealed any significant relationship (data not shown), except for CD247 expression. As shown in Fig. $4 D$, the last eight patients recruited were tested, and a significant correlation was found between ADCC variation (before versus after therapy) and the CD247 expression intensity variation on NK cells (before versus after therapy), but not on $\mathrm{T}$ cells (not shown). These latter findings suggest that the variation of potential ADCC observed with therapy may be related to parallel variations of CD247, the signal-transducing molecule for CD16 on NK cells.

Insights on the relationship between ADCC potential and the therapeutic outcome of short-term trastuzumab monotherapy in patients with operable breast cancer overexpressing Her2. The evaluation of clinical responses in terms of tumor reduction reported one complete pathologic response, four partial responses, and 12 patients with stable disease (Table 1). The most relevant association between ADCC and clinical response occurred for the patient with maximal ADCC activity (Fig. 3), who revealed pathologic complete response when residual tumor mass was analyzed after surgery. This patient underwent local irradiation after surgery, and remained free from disease after 5 years of follow-up. In the remaining cases, a significant degree of ADCC activity was commonly observed in patients with partial response (patients 2, 6, 10, and 13). After surgery, these patients received adjuvant treatments in accordance with standard practice guidelines. Patients 2 and 10 remained free from disease after near 5 years of follow-up; patient 6 developed bone metastases 13 months after surgery, and patient 13 was lost to follow-up after adjuvant treatment. As opposed to cases developing significant
Figure 2. Phenotypic modifications observed on effector lymphocytes at the final stages of trastuzumab-induced ADCC assay. Mononuclear cells and Her2-overexpressing target cells were cultured similarly to ADCC assay at 25:1 effector to target ratio, in the absence or presence of trastuzumab $(10 \mu \mathrm{g} / \mathrm{mL})$. At the end of the culture, cells were stained with the indicated mAbs and analyzed by multiparametric flow cytometry. Analysis was limited to the lymphocyte gate identified by forward versus side scatter profiles. Top dot plots, gating of lymphocytes analyzed in each histogram column, including CD3 ${ }^{-} \mathrm{CD}^{+} 6^{+} \mathrm{NK}$ cells, $\mathrm{CD}^{+} 6^{+} \mathrm{T}$ cells, and CD56 ${ }^{-} \mathrm{T}$ cells. Second through bottom rows, histograms of CD16, CD69, and CD107a, respectively. Thin and thick lines, cell phenotype in the absence and presence of trastuzumab, respectively. Data are from patient 14 and are representative of at least one experiment similarly conducted in each other patient.

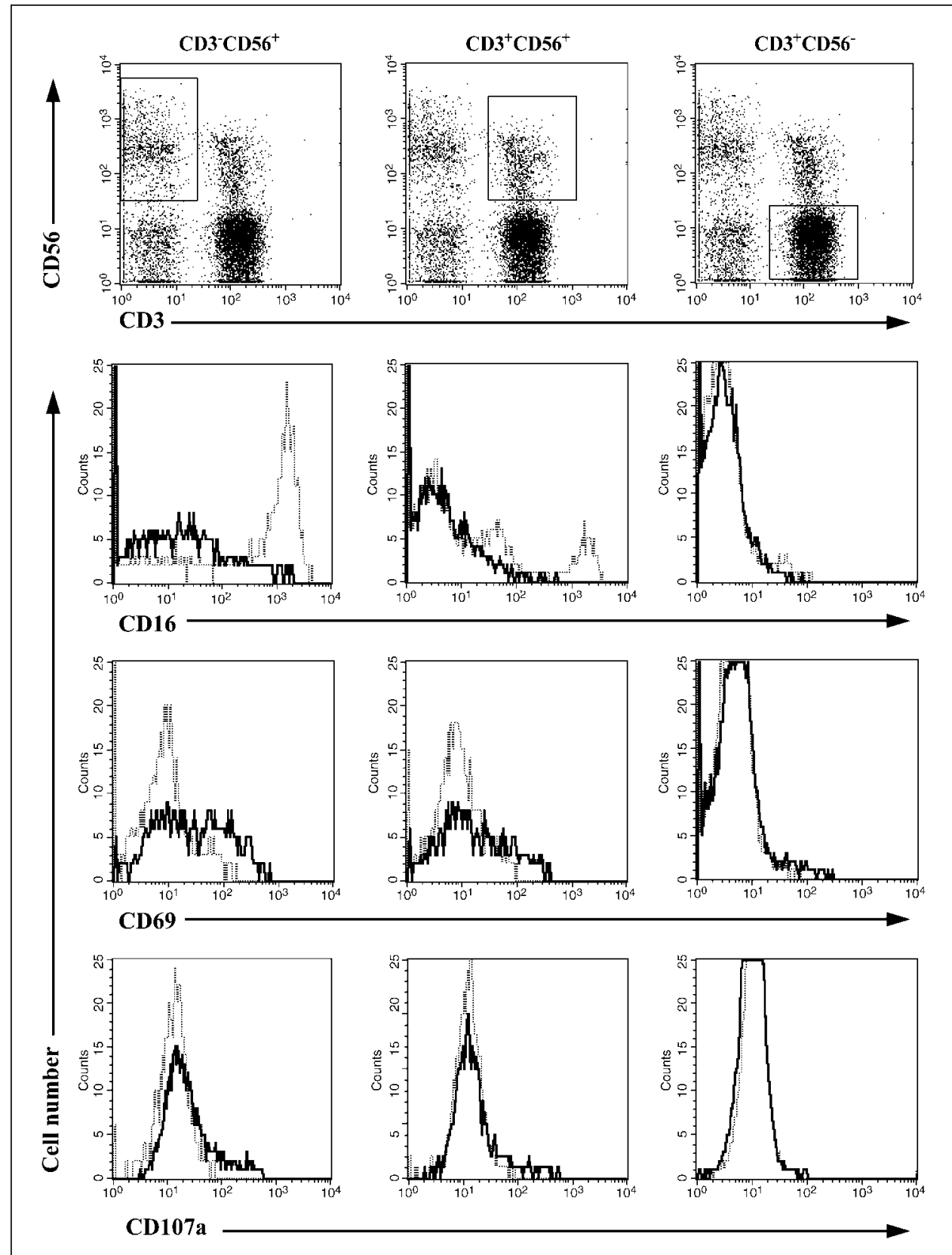




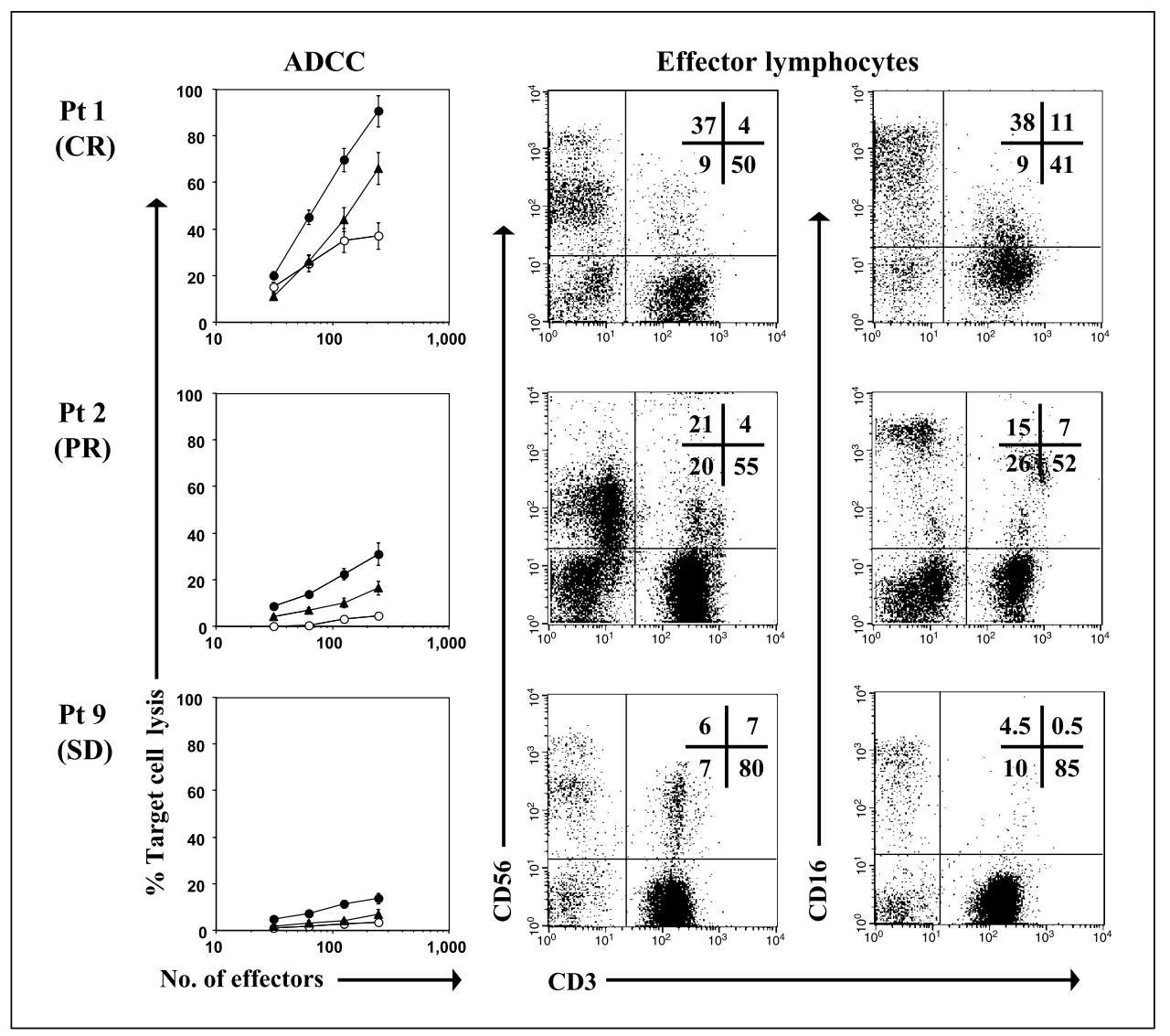

Figure 3. Relationship between ADCC induced by trastuzumab and patients' lymphocytes composition. Reported data on each row are from patients 1,2 , and 9 (top to bottom). Left column, the ADCC activity expressed as percent of target cell lysis at increasing effector to target ratios $(6.25,12.5,25$, and 50$)$ is reported; $O$, basal cytotoxicity in the absence of any trastuzumab source; $\boldsymbol{\Delta}$, basal ADCC in the presence of trastuzumab $(10 \mu \mathrm{g} / \mathrm{mL})$ added in vitro; $\bullet$, posttherapy ADCC in the presence of $20 \%$ autologous serum. Middle and right columns, composition of effector lymphocytes corresponding to each patient shown in the left column.

ADCC, none of the three unresponsive patients (patients 4, 8, and 18) plus another patient with very low ADCC (patient 9; Fig. 3) showed evidence of significant tumor regression (Table 1). As a result, the emerging relationship suggests a correlation at the upper and lower limits as in one case extremely high ADCC was associated with effective antitumor response, whereas extremely low ADCC was associated with ineffective antitumor responses.

\section{Discussion}

This study reports the analysis of ADCC induced by trastuzumab in a group of patients with operable breast cancer overexpressing Her2. Analysis was carried out on PBMC isolated from patients once therapy was under way, without interference deriving from other previous or concomitant antineoplastic therapies. Under these conditions, some patients were unable to acquire significant ADCC, whereas most patients developed low-to-intermediate ADCC levels, and one outstanding case showed maximal activity. This unexpected extreme variability was further dissected as the sum of cellular and humoral factors to clarify the origins of such heterogeneity and the importance of parameters influencing the clinical efficacy of ADCC by trastuzumab.

The influence of humoral factors on ADCC can involve several aspects. First of all, ADCC is related to $\mathrm{mAb}$ concentration in a dose-dependent manner (21). In addition, various soluble elements are known to inhibit ADCC in vivo. Endogenous IgGs can compete with IgG mAbs for binding to various classes of Fc $\gamma$ Rs, in particular with Fc $\gamma$ RIII $(15,31,32)$. Moreover, shedded Fc $\gamma$ RIII can also act as a decoy element partially neutralizing the Fc part of mAbs and other IgGs (33). Finally, Her2 can also be shedded in plasma in patients with breast cancer expressing high levels of this (34). All of these soluble elements may limit the productive interaction between FcyRIII (CD16) and the Fc portion of mAb bound to tumor cells. To take into account these potential effects and reproduce in vitro the ADCC activity developed in vivo by each patient, we conducted functional assays in the presence of a substantial concentration of complement-inactivated autologous serum harvested at the same time as the effector cells. In these conditions, we found that even when further adding trastuzumab at a concentration sufficient to restore maximal cell lysis (15), ADCC intensity was not modified in a significant manner (Fig. $1 B$ ). These results indicated that trastuzumab concentration under therapy was not inadequate and also suggested that other soluble inhibitory factors were probably not accountable for low-to-absent ADCC induction observed in some patients.

The evaluation of the cellular contribution to $\mathrm{ADCC}$ in patients under $\mathrm{mAb}$ therapy is complex because of many cell types expressing FcyRs that can engage mAbs of the IgG class (16-19). Through the analysis of PBMC, we have found indirect but clear evidences that $\mathrm{CD}^{+} 6^{+} \mathrm{CD} 56^{+} \mathrm{NK}$ and $\mathrm{T}$ lymphocytes, both of which are specialized for killing, were involved in ADCC (Figs. 2 and 3). The individual heterogeneity of ADCC was significantly dependent on the quantity of $\mathrm{CD} 16^{+} \mathrm{CD} 56^{+}$lymphocytes among PBMC (Figs. 3 and $4 A$ and $B$ ). Another level of individual heterogeneity was related to the killing efficiency of $\mathrm{CD}^{+} 6^{+} \mathrm{CD} 56^{+}$lymphocytes and possibly to their Fc $\gamma$ RIIIa 158 V/F polymorphism (Fig. 4C). Such a relationship between Fc $\gamma$ RIIIa $158 \mathrm{~V} / \mathrm{F}$ polymorphism and killing efficiency for $\mathrm{ADCC}$ is consistent with previous studies showing an increased affinity for IgG1 mAbs by subjects bearing Fc $\gamma$ RIII 158 
VV alleles $(20,21,35)$. However, the overall effect of killing efficiency on whole ADCC, as opposed to the quantity of $\mathrm{CD} 16^{+} \mathrm{CD} 56^{+}$lymphocytes, was not substantial in this limited cohort because patients with the highest ADCC (including patient 1) were distinct from those with the highest killing efficiency (including patients 7 and 17), and vice versa for low responders. Ultimately, the whole $\mathrm{ADCC}$ response was the product of the quantity and efficiency of $\mathrm{CD} 16^{+} \mathrm{CD} 56^{+}$lymphocytes, but the influence of the quantity appeared more relevant than efficiency. Such influence of the effector cell number might depend on the peculiar protocol, which included patients with unaffected immune system, whereas killing efficiency may play a more relevant role in patients rendered lymphopenic by cytotoxic agents because of advanced disease. Nevertheless, these data draw attention to the relevance of effector cell numbers when considering the ADCC mechanism of action.

The prediction of ADCC intensity by preliminary assay before therapy may represent an instrument to classify patients more or less likely to benefit from the ADCC mechanism of action mediated by trastuzumab. In spite of the typical variability intrinsic to most functional cell assay, we observed that it was possible to identify at least patients with extremely high or low susceptibility toward developing ADCC responses after short-term therapy with trastuzumab (Fig. $1 C$ ). These findings suggest that an integrated evaluation of ADCC along with the quantification of effector $\mathrm{CD}_{16}{ }^{+} \mathrm{CD} 56^{+}$lymphocytes and Fc $\gamma \mathrm{RIII}$ polymorphisms should provide a way in the future to estimate patients' tendencies to develop potential ADCC responses in vivo under therapy with trastuzumab.

The role of $\mathrm{ADCC}$ or other indirect immune-mediated mechanisms in clinical activity of trastuzumab is still being debated and it is difficult to definitely prove. However, next to elegant demonstrations in animal models, recent clinical studies have also provided preliminary evidences that NK cells can be activated (36) and recruited $(12,13)$ by trastuzumab therapy, whereas other emerging studies are ready to report a correlation between clinical outcomes and Fc $\gamma R$ s polymorphisms in advanced stages of the disease (37). Hence, the question probably is not if ADCC can be triggered against established breast cancer, but rather when and how much ADCC can be efficacious at the clinical level, or at which preferential stages of the disease, or with which drug combination, and so forth. In this report, we confirmed and extended our previous findings (12) on the correlation existing between ADCC and response to trastuzumab in the preoperative setting. It should be noted that this setting may be considered favorable to document the specific contribution from the ADCC mechanism because the immune system mechanisms are still intact, and because the tumor regression seen after such a short treatment is more likely related to cytocidal killing-dependent event than to cytostatic events by trastuzumab. Under these circumstances, the potential for ADCC expressed by PBMC was extraordinarily high in a patient showing pathologic complete response, but it was absent
Figure 4. Relationships between trastuzumabinduced ADCC and its cellular and molecular mediators. $A$, correlation between percentages of $\mathrm{CD}^{+} 6^{+} \mathrm{CD} 16^{+}$effector cells among PBMC and $A D C C$ induced by in vitro addition of trastuzumab in patients before therapy. Rho and $P$ values refer to nonparametric Spearman correlation test. $B$, correlation between percentages of $\mathrm{CD}^{+} 6^{+} \mathrm{CD} 16^{+}$effector cells among PBMC and ADCC in the presence of $20 \%$ autologous serum in patients after therapy. Rho and $P$ values refer to nonparametric Spearman correlation test. $C$, distribution of target cell number lysed by $1,000 \mathrm{CD}^{2} 6^{+} \mathrm{CD} 16^{+}$effector cells among patients classified according to CD16 V/F polymorphism at 158 amino acid position. Horizontal bars, median values for each group. $D$, relationship between the ratio of CD247 expression intensity before/after therapy in eight consecutive patients tested (patients 11-18), and the ratio of ADCC before/ after therapy. Rho and $P$ values refer to the nonparametric Spearman correlation test.

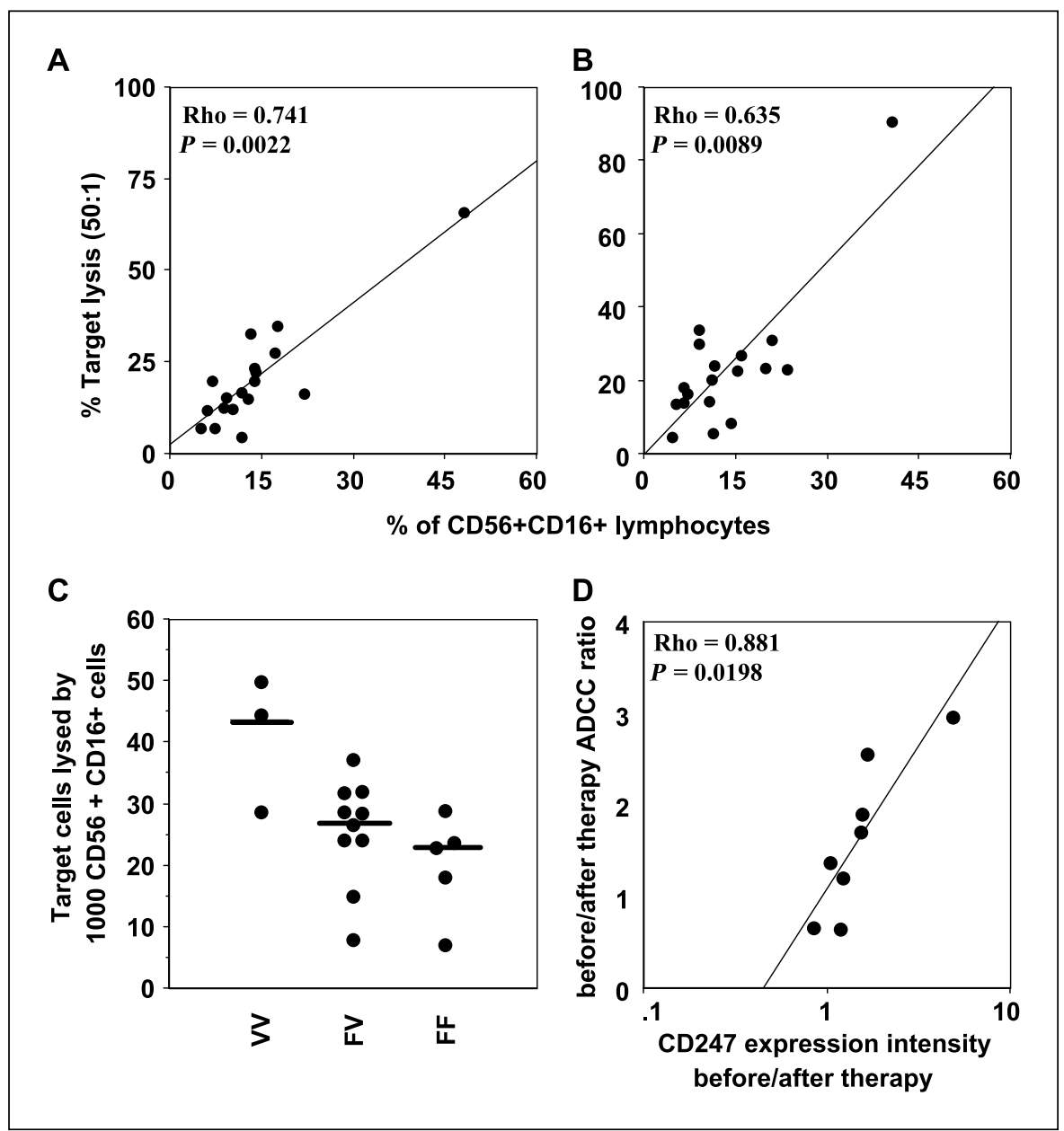




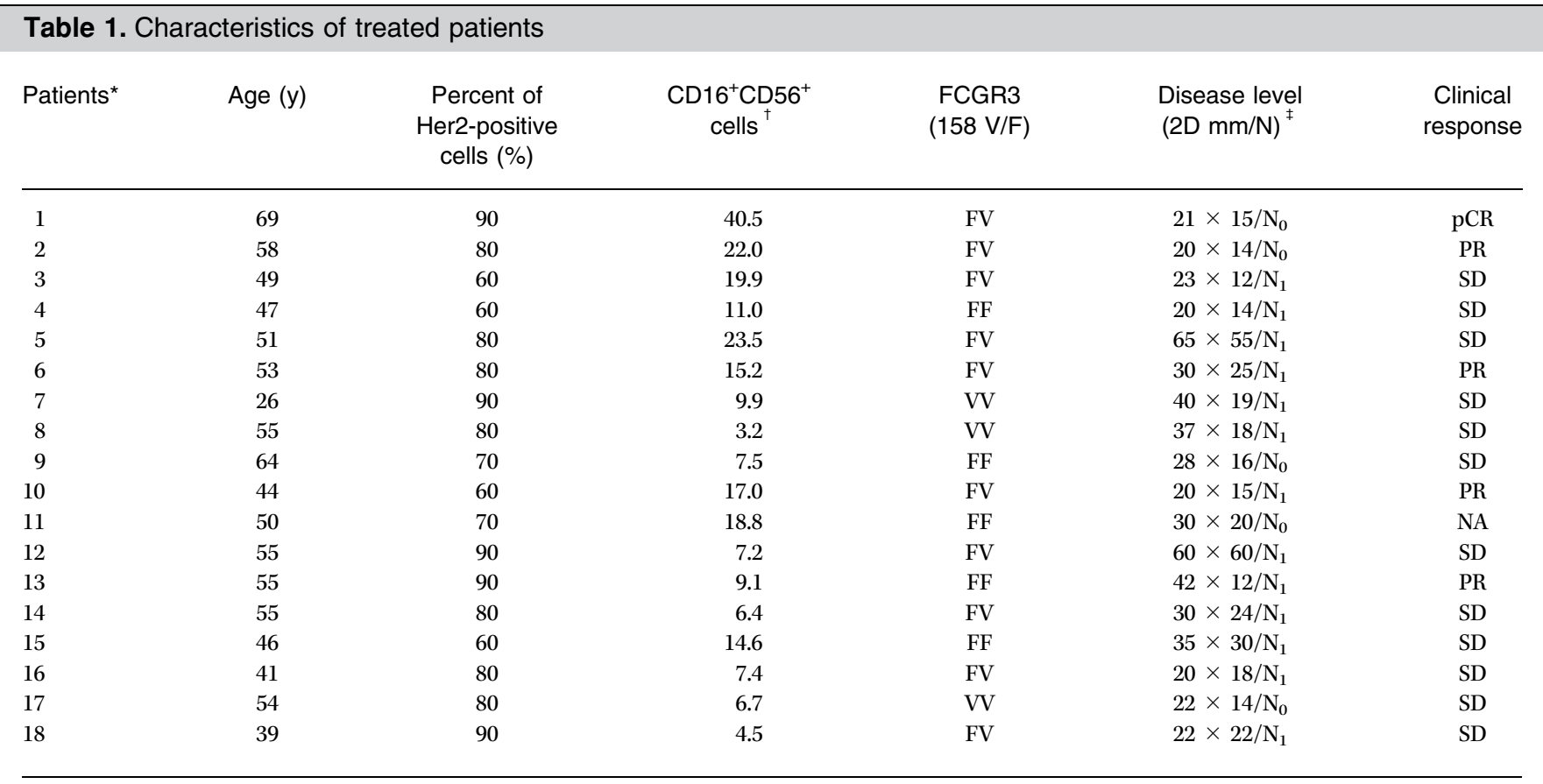

Abbreviations: pCR, pathologic complete response; PR, partial response; SD, stable disease; NA, not available.

*Patients were numbered according to recruitment progression.

tPercentages refer to PBMC tested for ADCC assay.

$\ddagger$ Disease level refers to primary tumor mass (expressed in $\mathrm{mm}$ as evaluated in bidimensional radiologic/ultrasound examination) and to lymph node involvement $(\mathrm{N})$.

in three patients (patients 4, 8, and 18) and extremely low in another patient (patient 9), all of whom lacked evidences of significant tumor regression (Table 1). Moreover, although many patients clustered their ADCC quite narrowly and intermediate ADCC activity was scarcely predictive for clinical activity, it should be noted that all patients with significant antitumor response (Table 1) also had considerable ADCC (Fig. 1). In the end, the relationship was particularly evident not only for the patient showing extremely high ADCC, but also for the unfortunate but more common cases of absent or low ADCC activity. This latter observation raises the possibility that low ADCC may be at the origin of some cases of intrinsic primary resistance to trastuzumab.

In conclusion, this study indicates that the systemic potential to exert CD16-dependent ADCC, a lytic attack on antibody-targeted cells, in patients with established breast cancer under treatment with trastuzumab is highly heterogeneous, primarily dependent on the availability of functional effector cells, and fairly predictable in vitro within a short time from the beginning of therapy. By contrast, the availability of circulating trastuzumab should not be considered a limiting factor for ADCC when weekly administrations at common dosages are used. The coincidence of maximal ADCC with pathologic complete response and of low-to-absent ADCC with a lack of clinical response suggests that high or low ADCC can either add or limit trastuzumab clinical activity, respectively. These observations contribute to identifying patients with opposite susceptibilities to developing ADCC with short-term trastuzumab, to further clarifying the role played by ADCC in trastuzumab therapy, and possibly to optimizing the therapeutic efficiency of trastuzumab in breast cancer patients overexpressing Her2.

\section{Acknowledgments}

Received 6/7/2007; revised 8/30/2007; accepted 10/11/2007.

Grant support: Progetto di Ricerca Finalizzata 2003 del Ministero della Salute, Rome, Italy, and Associazione Italiana di Ricerca sul Cancro, Milan, Italy.

The costs of publication of this article were defrayed in part by the payment of page charges. This article must therefore be hereby marked advertisement in accordance with 18 U.S.C. Section 1734 solely to indicate this fact.

\section{References}

1. Burstein HJ, Winer EP. HER2 or not HER2: that is the question. J Clin Oncol 2005;23:3656-9.

2. Slamon DJ, Leyland-Jones B, Shak S, et al. Use of chemotherapy plus a monoclonal antibody against HER2 for metastatic breast cancer that overexpresses HER2. N Engl J Med 2001;344:783-92.

3. Piccart-Gebhart MJ, Procter M, Leyland-Jones B, et al. Trastuzumab after adjuvant chemotherapy in HER2 positive breast cancer. N Engl J Med 2005;353:1659-72.
4. Romond EH, Perez EA, Bryant J, et al. Trastuzumab plus adjuvant chemotherapy for operable HER2-positive breast cancer. N Engl J Med 2005;353:1673-84.

5. Mohsin SK, Weiss HL, Gutierrez MC, et al. Neoadjuvant trastuzumab induces apoptosis in primary breast cancers. J Clin Oncol 2005;23:2460-8.

6. Nahta R, Yu D, Hung MC, Hortobagyi GN, Esteva FJ. Mechanisms of disease: understanding resistance to HER2-targeted therapy in human breast cancer. Nat Clin Pract Oncol 2006;3:269-80.

7. Valabrega G, Montemurro F, Aglietta M. Trastuzumab: mechanism of action, resistance and future perspectives in HER2-overexpressing breast cancer. Ann Oncol 2007; 18:977-84.

8. Clynes RA, Towers TL, Presta LG, Ravetch JV. Inhibitory $\mathrm{Fc}$ receptors modulate in vivo cytotoxicity against tumor targets. Nat Med 2000;6:443-6.

9. Fleming GF, Meropol NJ, Rosner GL, et al. A phase I trial of escalating doses of trastuzumab combined with daily subcutaneous interleukin 2: report of cancer and leukemia group B 9661. Clin Cancer Res 2002;8: 3718-27. 
10. Repka T, Chiorean EG, Gay J, et al. Trastuzumab and interleukin-2 in HER2-positive metastatic breast cancer: a pilot study. Clin Cancer Res 2003;9:2440-6.

11. Parihar R, Nadella P, Lewis A, et al. A phase I study of interleukin 12 with trastuzumab in patients with human epidermal growth factor receptor-2-overexpressing malignancies: analysis of sustained interferon gamma production in a subset of patients. Clin Cancer Res 2004; 10:5027-37.

12. Gennari R, Menard S, Fagnoni F, et al. Pilot study of the mechanism of action of preoperative trastuzumab in patients with primary operable breast tumors overexpressing HER2. Clin Cancer Res 2004;10:5650-5.

13. Arnould L, Gelly M, Penault-Llorca F, et al. Trastuzumab-based treatment of HER2-positive breast cancer: an antibody-dependent cellular cytotoxicity mechanism? Br J Cancer 2006;94:259-67.

14. Menard S, Pupa SM, Campiglio M, et al. Apoptosis induction by trastuzumab: possible role of the core biopsy intervention. J Clin Oncol 2005;23:7238-40.

15. Preithner S, Elm S, Lippold S, et al. High concentrations of therapeutic IgGl antibodies are needed to compensate for inhibition of antibody-dependent cellular cytotoxicity by excess endogenous immunoglobulin G. Mol Immunol 2006;43:1183-93.

16. Carson WE, Parihar R, Lindemann MJ, et al Interleukin-2 enhances the natural killer cell response to Herceptin-coated Her2/neu-positive breast cancer cells. Eur J Immunol 2001:31:3016-25.

17. Lanier LL, Kipps TJ, Phillips JH. Functional properties of a unique subset of cytotoxic $\mathrm{CD}^{+} \mathrm{T}$ lymphocytes that express Fc receptors for IgG (CD16/Leu-11 antigen). J Exp Med 1985;162:2089-106.

18. Lefebvre ML, Krause SW, Salcedo M, Nardin A. Ex vivo-activated human macrophages kill chronic lymphocytic leukemia cells in the presence of rituximab: mechanism of antibody-dependent cellular cytotoxicity and impact of human serum. J Immunother 2006;29: 388-97.
19. Stockmeyer B, Beyer T, Neuhuber W, et al. Polymorphonuclear granulocytes induce antibody-dependent apoptosis in human breast cancer cells. J Immunol 2003;171:5124-9.

20. Koene HR, Kleijer M, Algra J, Roos D, von dem Borne $\mathrm{AE}$, de Haas M. Fc $\gamma \mathrm{RIIIa}-158 \mathrm{~V} / \mathrm{F}$ polymorphism influences the binding of IgG by natural killer cell $\mathrm{Fc}$ $\gamma \mathrm{RIII}$, independently of the Fc $\gamma \mathrm{RIIIa}-48 \mathrm{~L} / \mathrm{R} / \mathrm{H}$ phenotype. Blood 1997;90:1109-14.

21. Dall'Ozzo S, Tartas S, Paintaud G, et al. Rituximabdependent cytotoxicity by natural killer cells: influence of FCGR3A polymorphism on the concentration-effect relationship. Cancer Res 2004;64:4664-9.

22. Weng WK, Levy R. Two immunoglobulin G fragmen $\mathrm{C}$ receptor polymorphisms independently predict response to rituximab in patients with follicular lymphoma. J Clin Oncol 2003;21:3940-7.

23. Cartron G, Dacheux L, Salles G, et al. Therapeutic activity of humanized anti-CD20 monoclonal antibody and polymorphism in IgG Fc receptor Fc $\gamma$ RIIIa gene. Blood 2002;99:754-8.

24. Treon SP, Hansen M, Branagan AR, et al. Polymorphisms in FcyRIIIA (CD16) receptor expression are associated with clinical response to rituximab in Waldenstrom's macroglobulinemia. J Clin Oncol 2005; 23:474-81.

25. Farag SS, Flinn IW, Modali R, Lehman TA, Young D, Byrd JC. Fc $\gamma$ RIIIa and Fc $\gamma$ RIIa polymorphisms do not predict response to rituximab in B-cell chronic lymphocytic leukemia. Blood 2004;103:1472-4.

26. Lin TS, Flinn IW, Modali R, et al. FCGR3A and FCGR2A polymorphisms may not correlate with response to alemtuzumab in chronic lymphocytic leukemia. Blood 2005;105:289-91.

27. Therasse P, Arbuck SG, Eisenhauer EA, et al. New guidelines to evaluate the response to treatment in solid tumors. J Natl Cancer Inst 2000;92:205-16.

28. Fagnoni FF, Oliviero B, Giorgiani G, et al. Reconstitution dynamics of plasmacytoid and myeloid dendritic cell precursors after allogeneic myeloablative hematopoietic stem cell transplantation. Blood 2004;104:281-9. 29. Kono K, Ressing ME, Brandt RM, et al. Decreased expression of signal-transducing $\zeta$ chain in peripheral $\mathrm{T}$ cells and natural killer cells in patients with cervical cancer. Clin Cancer Res 1996;2:1825-8.

30. Bottcher S, Ritgen M, Bruggemann M, et al. Flow cytometric assay for determination of Fc $\gamma$ RIIIA- $158 \mathrm{~V} / \mathrm{F}$ polymorphism. J Immunol Methods 2005;306:128-36.

31. Mikulski SM, Billing R, Terasaki PI. Inhibition of effector cell function in human antibody-dependent cellular cytotoxicity by sera from cancer patients. J Nat Cancer Inst 1977;58:1485-7.

32. Iida S, Misaka $H$, Inoue $M$, et al. Nonfucosylated therapeutic IgGl antibody can evade the inhibitory effect of serum immunoglobulin G on antibodydependent cellular cytotoxicity through its high binding to Fc $\gamma$ RIIIa. Clin Cancer Res 2006;12:2879-87.

33. Huizinga TW, de Haas M, van Oers $\mathrm{MH}$, et al. The plasma concentration of soluble Fc- $\gamma$ RIII is related to production of neutrophils. Br J Haematol 1994;87: 459-63.

34. Mazouni C, Hall A, Broglio K, et al. Kinetics of serum HER-2/neu changes in patients with HER-2-positive primary breast cancer after initiation of primary chemotherapy. Cancer 2007;109:496-501.

35. Bowles JA, Weiner GJ. CD16 polymorphisms and NK activation induced by monoclonal antibody-coated target cells. J Immunol Methods 2005;304:88-99.

36. Roda JM, Parihar R, Magro C, Nuovo GJ, Tridandapan $\mathrm{S}$, Carson WE 3rd. Natural killer cells produce $\mathrm{T}$ cellrecruiting chemokines in response to antibody-coated tumor cells. Cancer Res 2006;66:517-26.

37. Musolino A, Naldi N, Bortesi B, et al. Immunoglobulin $\mathrm{G}$ fragment $\mathrm{C}$ receptor polymorphisms and response to trastuzumab-based treatment in patients with HER-2/neu-positive metastatic breast cance [abstract 4188]. 2007 AACR Annual Meeting, April 14-18, 2007, Los Angeles, CA. 


\section{Cancer Research}

\section{Elements Related to Heterogeneity of Antibody-Dependent Cell Cytotoxicity in Patients Under Trastuzumab Therapy for Primary Operable Breast Cancer Overexpressing Her2}

Stefania Varchetta, Nadia Gibelli, Barbara Oliviero, et al.

Cancer Res 2007;67:11991-11999.

Updated version Access the most recent version of this article at:

http://cancerres.aacrjournals.org/content/67/24/11991

Cited articles This article cites 36 articles, 20 of which you can access for free at:

http://cancerres.aacrjournals.org/content/67/24/11991.full\#ref-list-1

Citing articles This article has been cited by 27 HighWire-hosted articles. Access the articles at:

http://cancerres.aacrjournals.org/content/67/24/11991.full\#related-urls

E-mail alerts Sign up to receive free email-alerts related to this article or journal.

Reprints and To order reprints of this article or to subscribe to the journal, contact the AACR Publications Subscriptions Department at pubs@aacr.org.

Permissions To request permission to re-use all or part of this article, contact the AACR Publications Department at permissions@aacr.org. 